\title{
Localised Polymer Networks in Chiral Nematic Liquid Crystals for High Speed Photonic Switching
}

Chloe C. Tartan*, Patrick S. Salter, Martin J. Booth, Stephen M. Morris and Steve J. Elston*

Department of Engineering Science, University of Oxford, Parks Road, Oxford, OX1 3PJ, United Kingdom

E-mail: chloe.tartan@eng.ox.ac.uk, steve.elston@eng.ox.ac.uk

Keywords: liquid crystals, chiral, polymers, two-photon absorption, flexoelectro-optic effect

Self-assembled periodic structures based upon chiral liquid crystalline materials have significant potential in the field of photonics ranging from fast-switching optoelectronic devices to low-threshold lasers. The flexoelectro-optic effect, which is observed in chiral nematic liquid crystals (LCs) when an electric field is applied perpendicular to the helical axis, has significant potential as it exhibits analogue switching in 10-100 $\mu$ s. However, the major technological barrier that prohibits the commercial realisation of this electro-optic effect is the requirement of a uniform, in-plane alignment of the helix axis between glass substrates. Here it is shown that periodic polymer structures engineered in the nematic phase of a chiral nematic LC device using direct laser writing can result in the spontaneous formation of the necessary uniform lying helix (ULH) state. Specifically, two-photon polymerization is used in conjunction with a spatial light modulator so as to correct for aberrations introduced by the bounding glass substrates enabling the polymer structures to be fabricated directly into the device. The ULH state appears to be stable in the absence of an externally applied electric field and the optimum contrast between the bright and dark states is obtained using polymer structures that have periodicities of the order of the device thickness. 


\section{Introduction}

At present, the multi-billion dollar flat panel displays industry is dominated by Liquid Crystal (LC) technology. However, the main drawback with using LC technology is that the shortest response time that is achievable is of the order of milliseconds, which is limited by the viscoelastic response in nematic LCs. So far, there has not been widespread commercialisation of any of the fast-switching electro-optic effects that can be observed in LCs such as ferroelectric switching in the chiral smectic C phase or the flexoelectro-optic effect in the chiral nematic phase as both suffer from the fact that they are notoriously difficult to align uniformly, resulting in a poor optical contrast ratio. ${ }^{[1-4]}$ A solution to these alignment challenges would open up potential avenues for a fast-switching LC mode to be realised and may potentially enable field sequential colour generation thereby removing the dependency on colour filters. This could then lead to LCD screens with an improved brightness and colour gamut, or potentially lead to more advanced display technologies by resolving multiple images for the realization of multi-view autostereoscopic $3 \mathrm{D}$ displays. ${ }^{[5]}$

As stated, an electro-optic effect that has potential for next-generation fast-switching devices is the flexoelectro-optic (FEO) effect that is observed when a short pitch chiral nematic is aligned in a uniform lying helix (ULH) state, as illustrated in Figure 1, whereby the helical axis of the chiral nematic is aligned uniformly between two substrates. ${ }^{[3,4]}$ Under the application of an electric field perpendicular to the helix axis, an in-plane tilt $(\Phi)$ is induced in the optical axis that couples to the flexoelectric coefficients, which for small tilt angles is linear in the applied electric field, E, as shown in the following expression,

$$
\tan \Phi=(e p /(2 \pi k)) \mathrm{E}
$$


where $p$ is the pitch of the helix, $k=\left(K_{11}+K_{33}\right) / 2$ is the average elastic constant and $e=\left(e_{1}\right.$ $\left.e_{3}\right) / 2$ is the effective flexoelectric coefficient. Short-pitch chiral nematic LCs permit fast changes in the optical state leading to microsecond range response times given by,

$$
\tau=\gamma p^{2} /\left(4 \pi^{2} k\right)
$$

where $\gamma$ is the rotational viscosity. ${ }^{[4]}$ Conventional nematic LCDs are limited by the viscoelastic response leading to different 'on' and 'off' response times. The ULH response time in Equation 2 is the characteristic time it takes for $\Phi(\mathrm{E})$ to relax to its equilibrium value in both directions. ${ }^{[6]}$ Therefore, the FEO effect has potential to reduce the response times from milliseconds down to sub-milliseconds for next generation displays and optoelectronic devices.

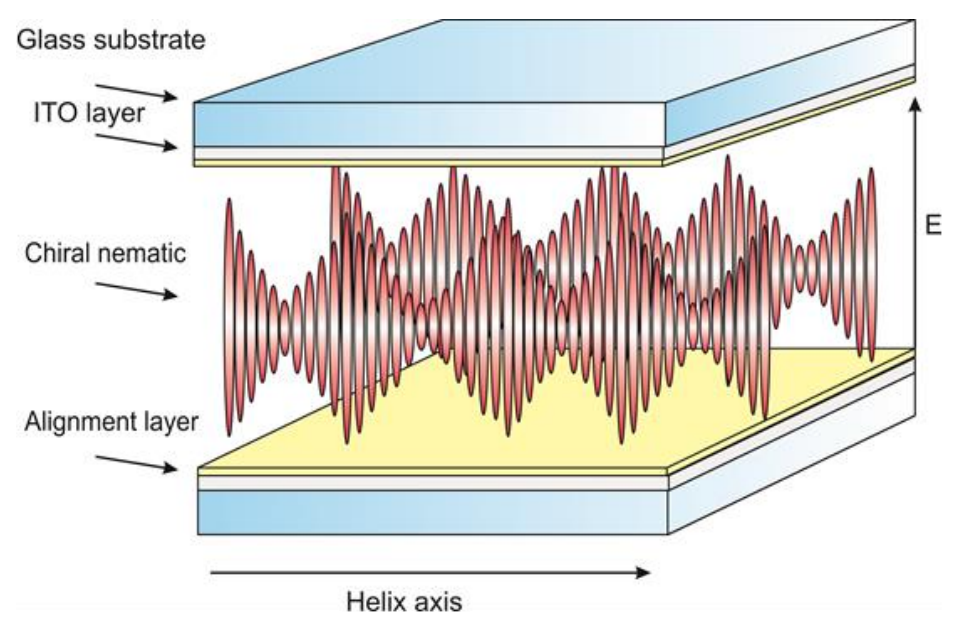

Figure 1. A schematic of the uniform lying helix state in a chiral nematic LC device.

The most challenging issue to overcome, however, is the fact that the ULH state is incompatible with strong planar and strong homeotropic alignments, becoming unstable when the chiral nematic is confined between spatially uniform aligning surfaces, such as a 
conventional glass cell. ${ }^{[7]}$ For planar anchoring at the substrates, the chiral nematic typically adopts a standing helix (Grandjean) alignment. It is well-known that applying an external electric field parallel to the helix axis of a chiral nematic sample with a positive dielectric anisotropy causes the helix to align in random orientations, known as the focal conic texture. However, a ULH geometry can be formed upon cooling from the isotropic phase in the presence of an applied electric field, but this is not stable in the absence of the field and therefore relaxes back to the lower energy Grandjean state upon removal of the electric field. $^{[7]}$

In an attempt to lock-in the inherently unstable ULH structure, one approach is to form polymer networks in LCs so as to stabilise the alignment. ${ }^{[8-10]}$ Through the addition of a suitable reactive mesogen (liquid crystalline monomer) and photosensitive initiator, the polymer network can be formed by exposure to ultraviolet light. In this case, the alignment is both stable in the absence of an electric field and reappears spontaneously upon thermal cycling from either the isotropic phase or heating from the crystalline solid phase. However, formation of the polymer network results in a reduction in the electro-optic performance of the device due to the rotation of the helix (quantified by the tilt angle) becoming inhibited by the bulk polymer network. In a paper by Broughton et al., it was shown that increasing the concentration of polymer resulted in a reduction in the tilt angle of the optic axis for a given electric field strength and a decrease in the response time. ${ }^{[9]}$ Therefore, this method leads to a trade-off between the reduction in response times that displays manufacturers are primarily interested in, and optimizing tilt angles so that next generation displays can be operated at low electric field strengths $\left(<5 \mu \mathrm{m}^{-1}\right)$. As the ULH response times are naturally fast, maximising the intensity modulation by exploring different methods that stabilise the molecular alignment is vital. 
Initially proposed by Patel and Meyer ${ }^{[11]}$, an alternative approach is to introduce a set of defects or periodic distortions at the ULH/substrate interface in order for the alignment to be locked-in without polymerizing the bulk LC. Komitov et al. have reported the fabrication of periodic anchoring conditions using hard-contact photolithography to pattern a photoresist and stabilise the ULH texture in the presence of an applied electric field. ${ }^{[12]}$ Weak homeotropic alignment has been shown to give a lower energy ULH relative to the Grandjean texture. However, the rotational symmetry at the substrate causes the lying helix alignment to collapse into domains due to the lack of directionality for the helix to form. Carbone et al. exploited this stable, low energy ULH texture occurring at the homeotropic substrate by breaking its rotational symmetry using two-photon absorption (TPA) laser scanning lithography to form periodic surface relief structures with a periodicity equal to the pitch of the bulk chiral nematic. ${ }^{[10]}$ In their study, the authors demonstrate a stable ULH even in the absence of an electric field, although a fairly poor contrast ratio is obtained between the bright and dark states of the device by commercial standards.

The ULH structure may also be aligned and stabilised through confinement in polymer channels. This has previously been implemented using holographic techniques such as POLICRYPS, but this can result in large areas of polymerised LC that inhibits the device's electro-optic performance. ${ }^{[13]}$ Here, we aim to maintain the FEO capacity while still aligning and stabilising the ULH through the creation of a periodic diffuse polymer network. Direct laser writing is used to selectively illuminate the LC layer to photo-activate the polymerisation. This can be done while a voltage, sufficient to unwind the helix, is applied across the device. Thus regions of diffuse network may be formed thereby locking in a homeotropic alignment allowing the ULH to form between them when the voltage is removed. It is shown that devices prepared in this manner have the potential to enhance the observed 
FEO switching, relative to conventional bulk polymerization techniques that can inhibit the electro-optic effect.

\section{Experimental Section}

The direct laser writing process uses two-photon absorption to create polymerized features that are confined in three dimensions to sub-micrometre sizes. The high resolution of this method is due to a combination of the non-linear absorption process and a threshold effect in the photo-polymerization. ${ }^{[14]}$ The addition of a spatial light modulator (SLM) within the optical system can correct for aberrations due to the refractive index mismatch as light passes through the specimen and substrate, making it possible to engineer polymeric photonic structures in-situ and with tight focal spots achieved through the use of high NA objectives. ${ }^{[15]}$

The commercial eutectic E7 nematic LC mixture was used as the host material, and has a birefringence and dielectric anisotropy of $\Delta \mathrm{n}=0.219$ and $\Delta \varepsilon=13.7$ at $\mathrm{T}=20^{\circ} \mathrm{C}$. The chiral nematic mixture consisted of $68.5 \mathrm{wt} \% \mathrm{E} 7,4.1 \mathrm{wt} \%$ of the high twisting power chiral dopant BDH1281 (Merck Chemicals Ltd.), 26.8 wt \% RM257 reactive mesogen (Merck Chemicals Ltd.) and $0.6 \mathrm{wt} \%$ IRG819 photoinitiator (Merck Chemicals Ltd.) for absorption in the UV range. A $5 \mu \mathrm{m}$ thick cell was used that has homeotropic alignment layers on the inner surfaces of the substrates along with indium tin oxide (ITO) layers so that the device can be electrically addressed. The cell was capillary filled with the pre-polymerised-LC mixture in the isotropic phase, before being cooled to room temperature for fabrication.

Figure 2 shows a schematic of the direct laser writing system. The light source was a Titanium Sapphire oscillator, emitting $100 \mathrm{fs}$ pulses at $790 \mathrm{~nm}$ (for the polymerization process, the absorption of two photons at $790 \mathrm{~nm}$ is equivalent to the absorption of one photon at 395 
$\mathrm{nm}$ ) with a repetition rate of $80 \mathrm{MHz}$ and a maximum power of $300 \mathrm{~mW}$. The beam is expanded and directed onto a phase-only nematic LC SLM (Hamamatsu X10789-02) which is subsequently imaged in a $4 f$ configuration onto the back aperture of the focussing objective lens. A blazed phase grating of period $0.5 \mathrm{~mm}$ (25 pixels) was displayed on the SLM, redirecting the incident laser beam. A pinhole, which was positioned in the Fourier plane of the SLM, only passed light that was steered by the SLM grating. A $20 \times$ Zeiss objective with a $0.5 \mathrm{NA}$ and working distance of $2 \mathrm{~mm}$ was used to focus light into the LC devices. The devices were mounted on an air bearing translation stage offering high precision motion over $100 \mathrm{~mm}$ in $x$ and $y$, and $3 \mathrm{~mm}$ in $z$ (Aerotech ABL10100). Polymer structures were engineered within the LC layer while the device was addressed by a $1 \mathrm{kHz}, \pm 42.5 \mathrm{~V}$ square wave (electric

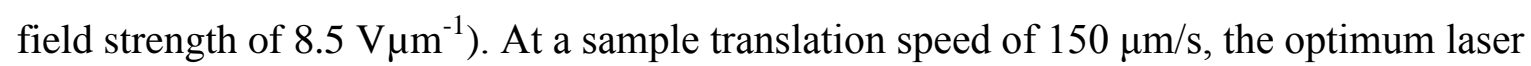
power was determined to be $75 \mathrm{~mW}$ to achieve narrow and uniform polymer walls.

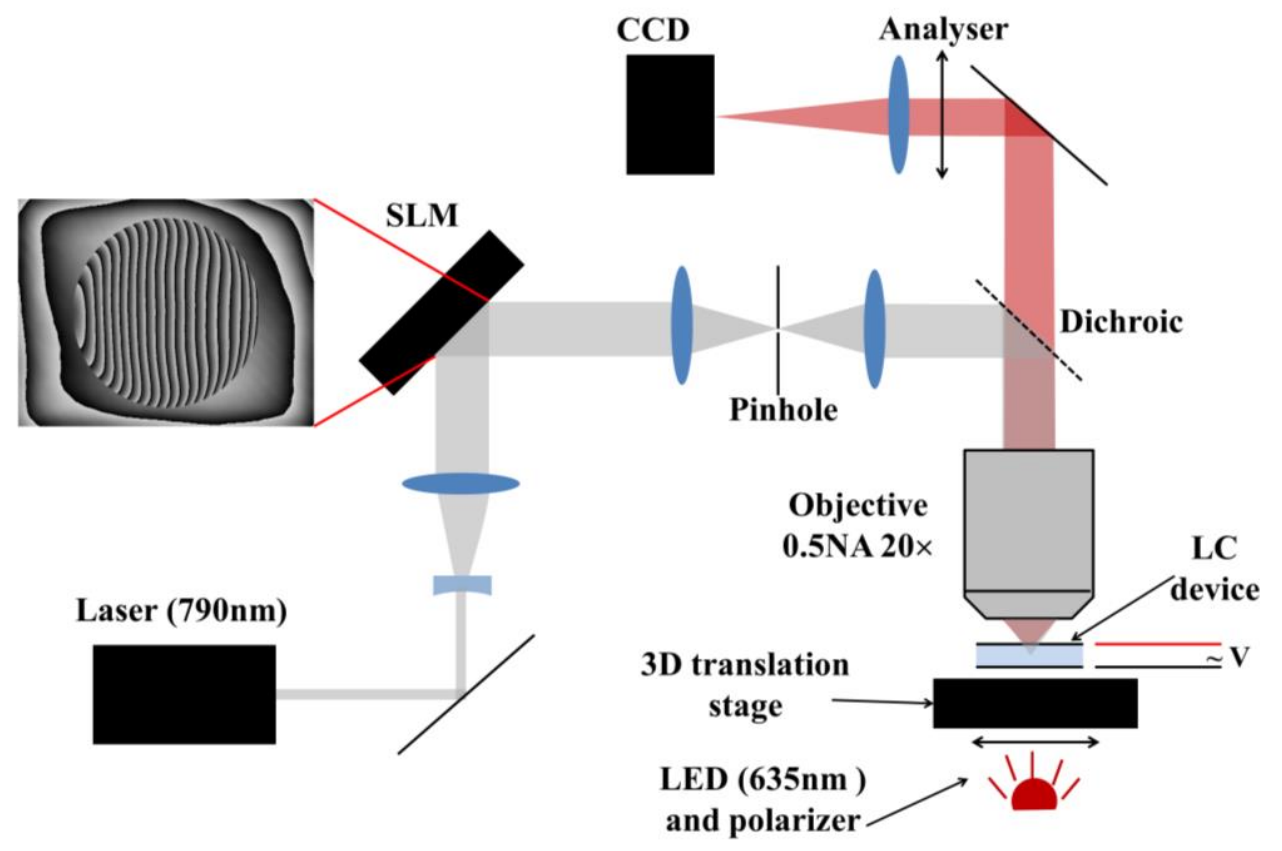

Figure 2. The TPA fabrication system with an SLM as the adaptive optics element. Ultrafast pulses of laser light are transmitted through various optical components, reaching a blazed phase grating that is projected onto the SLM to correct for aberrations in the sample due to the refractive index mismatch between the device's thick glass substrates and the LC layer. A high NA (0.5) objective lens is also used to fabricate structures at submicron resolutions and a 
red LED is used to illuminate the sample so that it can be monitored during the fabrication procedure.

For comparison, the electro-optic responses were measured from a ULH before photopolymerization and afterwards when the polymer networks were formed in the bulk of the device. This was achieved by cooling a planar device filled with the same chiral nematic LC-monomer mixture, to room temperature under the application of a $2 \mathrm{kHz}, \pm 27.5 \mathrm{~V}$ square wave and UV-curing the sample by exposure to a mercury vapour lamp at a distance of $10 \mathrm{~cm}$, emitting at $\lambda=365 \mathrm{~nm}$ and at a power density of $185 \mathrm{~mW} \mathrm{~cm}^{-2}$, for 12 seconds.

Tilt angles, $\Phi(\mathrm{E})$, were measured by initially rotating the device between crossed polarisers on an optical polarizing microscope so that the optic axis of the sample was angled at $22.5^{\circ}$ to the analyser, at the midpoint of the linear regime. Under the application of a moderate electric

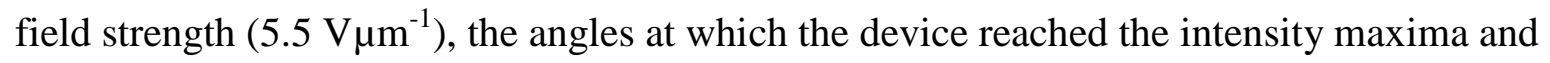
minima were noted, whereby the difference between the two is equivalent to $2 \Phi$. The same electric field strength was applied across all the devices in order to make a fair comparison between the devices; as the electric field is directly proportional to the tilt angle according to the relation in [1], a reduction in the flexoelectric coefficients would result in a larger field required to induce the same tilt angle, for all other factors remaining equal. It is worth noting that the electric field applied to measure the tilt angles was well below the critical electric

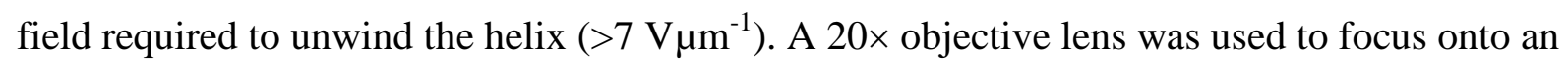
equivalent area in each device and a constant intensity of light from the microscope's halogen bulb was used to illuminate the samples, in order to make a fair comparison between the electro-optic behaviour of the three devices. A yellow colour filter was also included in the optical polarizing microscope to prevent further polymerization of the samples by exposure to the bulb. 


\section{Results and Discussion}

The fabrication process used to write the polymeric walls with varying periodicities, using the $200 \mathrm{~mW}$ Ti:S laser across regions of $0.2 \mathrm{~mm}$ by $1 \mathrm{~mm}$, can be seen in the schematic of Figure 3. A homeotropic device of $5 \mu \mathrm{m}$ thickness was filled with a chiral nematic LC-monomer mixture that had a pitch of $p \sim 400 \mathrm{~nm}$. Polymer walls were engineered in the nematic phase by exploiting the positive dielectric anisotropy of the LC mixture and unwinding the helix

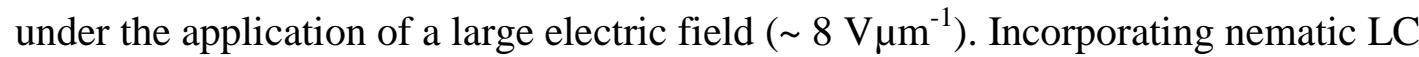
molecules into the network breaks the degeneracy of the homeotropic substrate and the fabrication of polymer walls that extend across the bulk of the device breaks the rotational symmetry of the homeotropic substrates in order to exploit the energetically favourable ULH state.

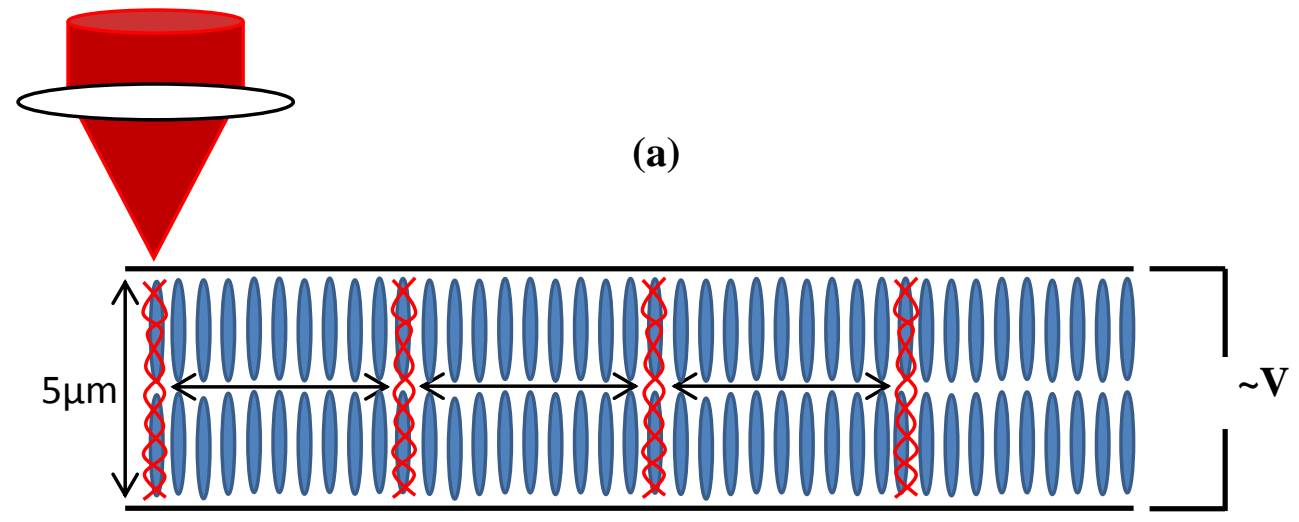

(b)

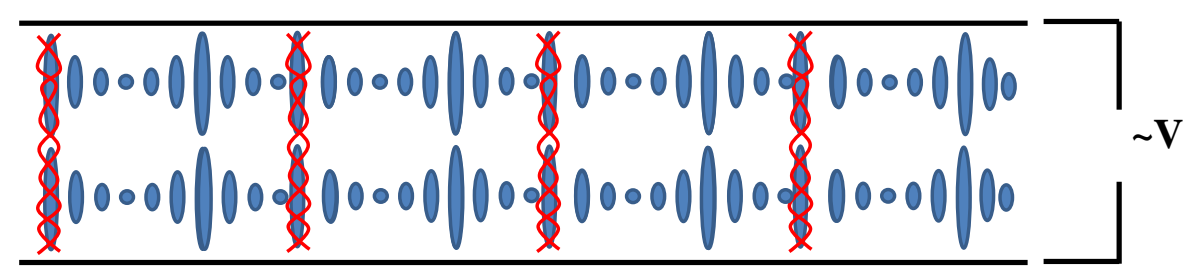

Figure 3. A schematic of the fabrication process showing the formation of the polymer network structures while the chiral nematic LC device with homeotropic boundaries is 
initially in (a) the nematic phase, whereby light from the laser (indicated by the red triangle) is used to write periodic polymer walls of varying periodicities under the application of an external electric field and at an optimal writing speed of $150 \mu / \mathrm{s}$. The energetically favourable ULH alignment forms in (b) the chiral nematic phase upon reduction of the applied electric field, whereby the helical axis tends to lie down in between the homeotropic polymer walls.

Polymer walls of $\sim 1 \mu \mathrm{m}$ thickness were engineered at varying periodicities and orientations in the chiral nematic LC device using the direct laser writing apparatus. Figure 4 shows an example image of the polymer structures taken between crossed polarisers using the built-in optical microscope of the direct laser writing experiment. As the electric field is gradually wound down into the chiral nematic phase, regions between the walls show the formation of chiral nematic fingers, with good optical extinction between crossed polarisers. In contrast, for the remaining areas of the device that do not consist of the periodic polymer walls, the images indicate that a focal conic texture has been formed.

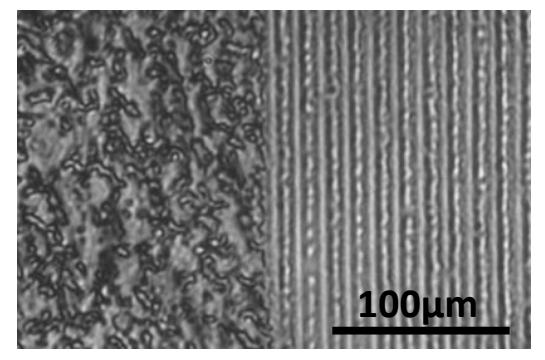

Figure 4. Image taken between crossed polarizers on the built-in polarizing optical microscope after the TPA fabrication process ( $100 \mathrm{fs}$ pulses at $\lambda=790 \mathrm{~nm}$ with a power of 2 $\mathrm{W}$ and a repetition rate of $1 \mathrm{kHz}$ ) of the polymer walls in a $5 \mu \mathrm{m}$ thick homeotropic device filled with the chiral nematic LC-monomer mixture. The device, with an electrode area of 1 $\mathrm{cm}^{2}$, shows the formation of chiral nematic fingers (bright stripes) in between the homeotropic polymer walls (dark stripes), while the surrounding LC regions appear to be a focal conic texture (random alignment of the helices).

Evidently, the polymer walls appear dark between crossed polarisers, suggesting that the nematic director has indeed been locked-in to a homeotropic alignment by the polymer network. This is because the polymer walls were fabricated in the homeotropic nematic state with the application of an electric field that was greater than that required to unwind the helix of the chiral nematic phase. It is unlikely that these dark stripes could be an indication of the 
isotropic phase (potentially due to the LC being melted during the fabrication process) as any unpolymerized LC would flow into such regions. The formation of these arrays of polymer walls is also supported by Scanning Electron Microscopy images. An example image for one particular periodicity is shown in Figure 5 with polymer walls that appear to be $\sim 2 \mu \mathrm{m}$ thick and spaced at $10 \mu \mathrm{m}$.

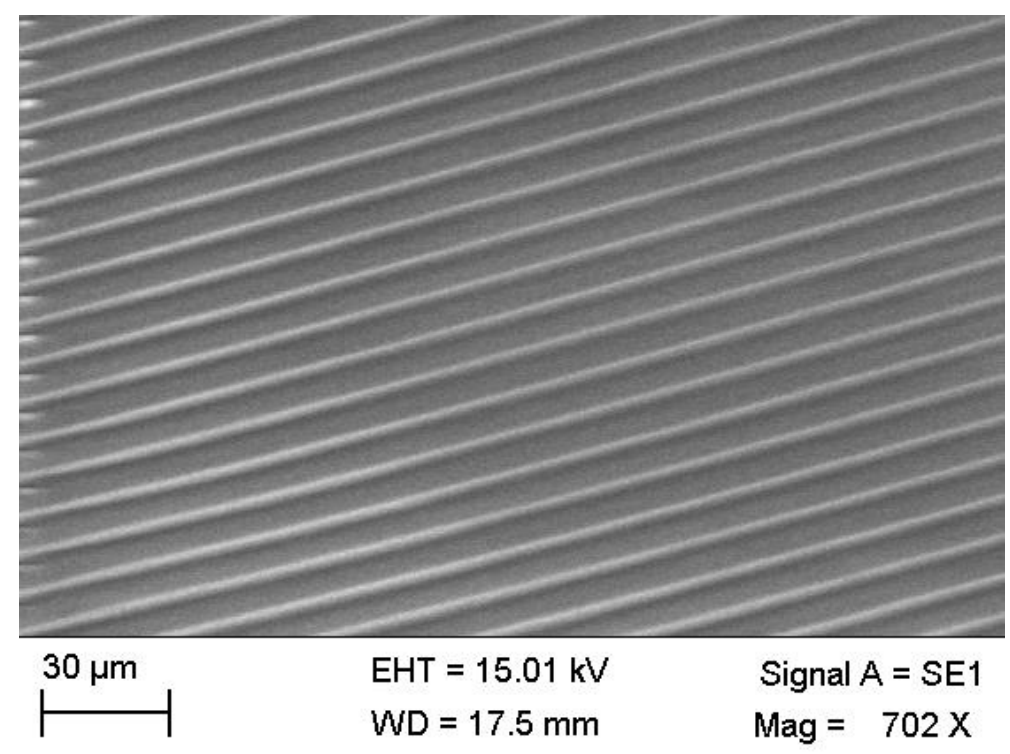

Figure 5. SEM image obtained using a secondary electron detector at an electron beam voltage of $15 \mathrm{kV}$ and a working distance of $17.5 \mathrm{~mm}$. The image shows periodic polymer walls (bright stripes) fabricated by a direct laser writing process.

Figure 6 shows images of the polymer LC structures with $10 \mu \mathrm{m}, 7.5 \mu \mathrm{m}, 5 \mu \mathrm{m}$ and $2.5 \mu \mathrm{m}$ periodicities taken between crossed polarizers on a polarizing optical microscope. All the structures remained stable in the absence of the externally applied electric field and upon thermal cycling. A Berek compensator revealed the helical axis to be perpendicular to the polymer walls. The regions of LC or 'microchannels' between the $10 \mu \mathrm{m}$-spaced polymer walls show poor contrast between the dark and bright states, where the device is angled at $0^{\circ}$ and $45^{\circ}$ to the analyser, respectively. There appears to be some alignment in regions near to the homeotropic polymer walls, however, the focal conic texture dominates throughout the microchannels. Decreasing the spacing between the polymer network structures to $7.5 \mu \mathrm{m}$ 
shows a marked improvement in the ULH alignment as well as the contrast between the two states. However, there still remains some focal conic alignment in the central regions of the channels. Optimum contrast (relative to the other periodicities shown in Figure 6) is observed between both bright and dark states at a periodicity of $5 \mu \mathrm{m}$, whereby the ULH state dominates most of the regions between the polymer walls. When the periodicity is decreased even further down to $2.5 \mu \mathrm{m}$ there is little to no transmission in the bright state, suggesting that the polymer network has diffused across the microchannels and locked-in the homeotropic molecular alignment.

We believe that the alignment of the chiral LC molecules in the nematic phase, combined with the nematic reactive mesogen, forces the helix axis to lie in the plane of the device. Figure 6 reveals that the polymer network is prone to some diffusion irrespective of the wall periodicities. The walls can be interpreted as sparsely cross-linked polymer chains that are partially diffuse across the microchannels thus encouraging the helix to lie down across the rest of the channel. Nevertheless, the SEM images do appear to show that, even though there is some diffusion, the walls are quite well defined. In the case of a short pitch chiral nematic LC with a $p=400 \mathrm{~nm}$, a $2.5 \mu \mathrm{m}$ periodicity of the walls results in approximately 6 pitches of the helix between each set of walls. For this number of pitches, the network is sufficiently dense between the lines of the walls, which are estimated to be $\sim 1 \mu \mathrm{m}$ in width on the optical polarizing microscope and appear to be closer to $2 \mu \mathrm{m}$ on the SEM, thereby leading to homeotropic nematic alignment of the LC everywhere. Therefore, this result indicates that the network diffuses roughly $1-2 \mu \mathrm{m}$ either side of the polymer walls. A $5 \mu \mathrm{m}$ periodicity leads to the formation of approximately 12 pitches of the helix, which from the optical images forms a well aligned ULH as the gap between the polymer walls is large enough for the chiral nematic phase to form, while increasing the periodicity further incorporates random orientations of the helical axis. 
Although the $5 \mu \mathrm{m}$ periodicity forms the optimum ULH alignment relative to the other periodicities shown in this study, the LC alignment overall results in a relatively low contrast ratio, as is evident from the microscope images. For example, stripes can still be seen at optical extinction, while the maximum transmission setting in the bright state is hampered by the black homeotropic polymer regions that cover almost $20 \%$ of the device area, although this may appear larger due to the diffusion of the polymer network between the walls that has previously been discussed. Nevertheless, this study is intended as a proof-of-concept and we believe that the alignment could be greatly improved through the careful selection of the reactive mesogen mixtures and the liquid crystalline host.

The nematic LC host, E7, also has an effect on the observed FEO response, which is limited by the dielectric coupling that tends to unwind the helix; thus, more careful selection of the LC host would be beneficial. Developments in recent years have led to new materials (bimesogenic compounds) that exhibit low dielectric anisotropy. These bimesogens extend the linear regime of the electro-optic effect and possess large flexoelastic ratios $(e / k)$ thus ensuring that full intensity modulation can be achieved with relatively low electric field strengths (e.g. 1- $2{\left.\mathrm{~V} \mu \mathrm{m}^{-1}\right) .}^{[16-18]}$ However, the proposed fabrication technique of the polymer walls does require some dielectric coupling in order for the polymer walls to be written in the nematic phase that occurs when the helix is unwound under an externally applied field. In this instance, a dual frequency LC compound ${ }^{[19,20]}$ may be a more suitable choice of material, whereby the polymeric structures could be fabricated in the positive dielectric anisotropy regime normally occurring at low frequencies when the sample is at room temperature. Subsequently, the device could be operated around the cross-over frequency $(\Delta \varepsilon \rightarrow 0)$ in order to maximise the FEO performance post-fabrication. 

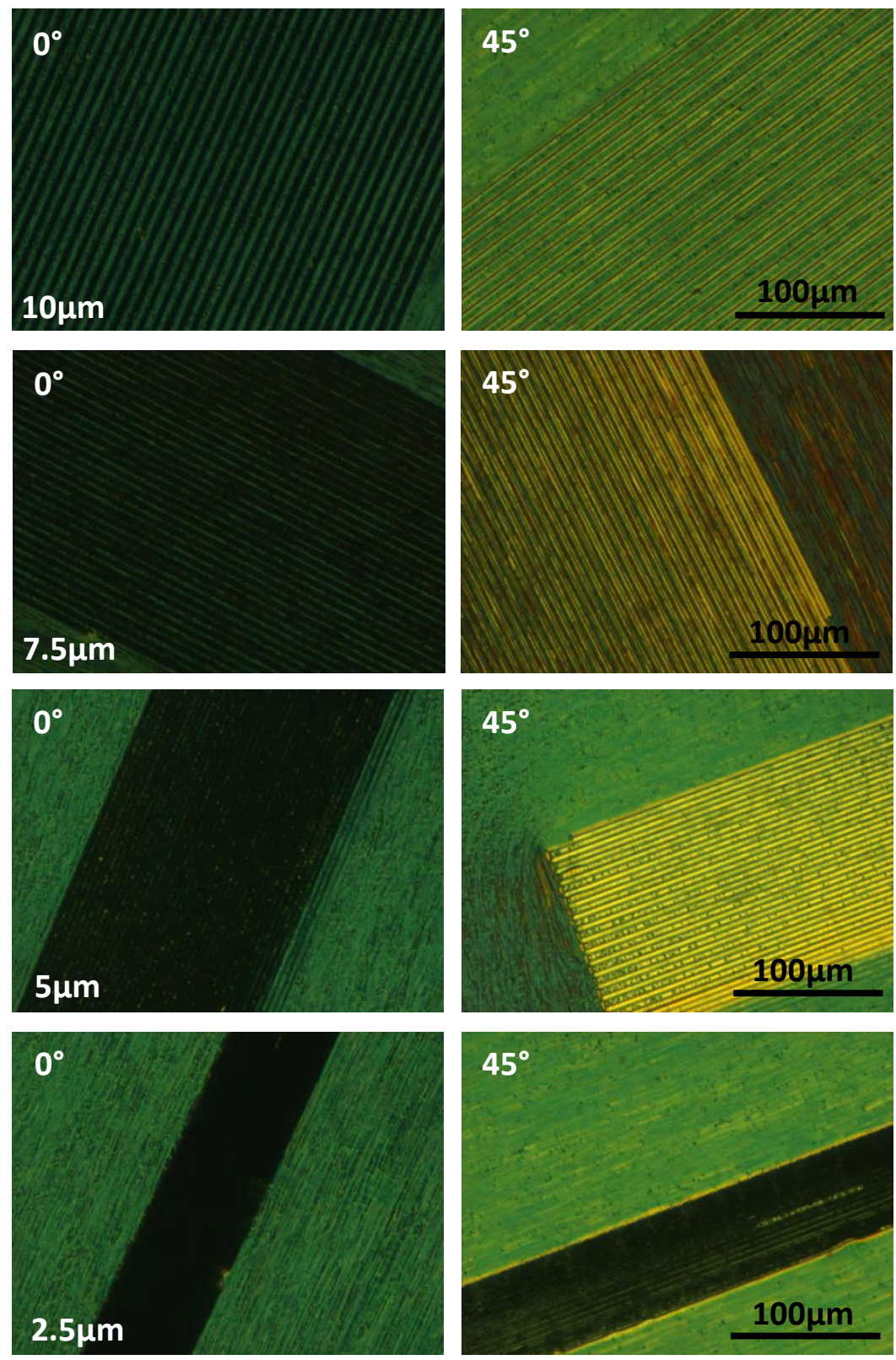

Figure 6. Optical images taken between crossed polarizers at optical extinction $\left(0^{\circ}\right)$ and maximum transmission $\left(45^{\circ}\right)$ of the devices with polymer walls spaced at $10 \mu \mathrm{m}, 7.5 \mu \mathrm{m}, 5$ $\mu \mathrm{m}$ and $2.5 \mu \mathrm{m}$. The structures were fabricated in a $5 \mu \mathrm{m}$-thick homeotropic device filled with the chiral nematic-monomer mixture. The devices, with an electrode area of $1 \mathrm{~cm}^{2}$, show homeotropic polymer walls (dark stripes) separated by channels of chiral nematic LC (bright stripes), while the surrounding LC regions appear to be a focal conic texture (random alignment). An improvement in the LC alignment can be seen as the spacing of the structures is decreased to be on the order of the device thickness.

The electro-optic responses from a planar aligned device in the ULH state before and after bulk polymerization are shown in Figure 7(a) and Figure 7(b), respectively. Weak modulation in the transmission is detected through the polymer-stabilised device; an initial pre-cure tilt 
angle of $\varphi=3.35^{\circ}$, obtained by rotating the device in the linear regime between the transmission maxima and minima, falls to $\varphi=0.13^{\circ}$ after polymerization under the application of a $2 \mathrm{kHz}, \pm 27.5 \mathrm{~V}$ square wave. This large reduction in the switching angle is also accompanied by a fall in the LC response time, from $\tau=100 \mu$ s before curing to $\tau=12$ $\mu \mathrm{s}$ after polymerization in the bulk. In contrast, Figure 7(c) shows that the fabrication of the polymer walls does not reduce the tilt angle by as large amount $\left(\varphi=1.73^{\circ}\right)$ when compared with that observed for bulk polymerization of the ULH alignment $\left(\varphi=0.13^{\circ}\right)$. Furthermore, the formation of these polymer walls ensures that the ULH alignment spontaneously recovers upon thermal cycling across the crystalline solid to chiral nematic and isotropic to chiral nematic phase transitions. It is also worth noting that the electric field applied here is well below the critical electric field required to unwind the helix $\left(>7 \mathrm{~V}^{-1}\right)$, yet high enough to obtain a measurable response from the device polymerized in the bulk.

The optical images presented in Figure 6 showing the bright and dark states as the device is rotated between crossed polarisers, combined with the electro-optic data presented in Figure 7(c), suggest that the LC alignment has formed a ULH texture. The modulated light intensity detected through the device with the $5 \mu \mathrm{m}$-spaced polymer walls, under the application of a $500 \mathrm{~Hz}, \pm 27.5 \mathrm{~V}$ square wave, shows a significant increase in the transmission modulation amplitude compared with that presented in Figure 7(b). Furthermore, the measured tilt angle from the device consisting of polymer walls was found to be $\varphi=1.73^{\circ}$, which is more than twenty times greater than that obtained from the device that was polymerized in the bulk (c.f. Figure 7(b)). In terms of the response time, the device consisting of the polymer walls appears to be of a similar order of magnitude (i.e. $~ 100 \mu$ s) to that recorded before polymerization. A response time of $\sim 100 \mu$ s observed from the data in Figure $7(\mathrm{~b})$, which is equivalent to that observed from the pre-cure ULH texture. However, the diffusion of the polymer network either side of the walls restrains the LC molecules leading to almost a $50 \%$ reduction in the tilt 
angle compared with the non-polymerized state even though the structured polymer walls alone occupy less than $20 \%$ of the device area.

The apparent tilt angle for the sample consisting of the polymer walls was calculated from the electro-optic response that was recorded over an area comprising both bright and dark stripes. As a result, this could mean that there is a locally varying electric field-induced tilt between the walls due to variations in the network density within these regions. Moreover, the high concentration of reactive mesogen in the mixture could give significant changes in the FEO response between the walls compared to that before polymerization. A potential method to limit diffusion of the polymer network into the LC channels could involve the addition of another monomer to the initial chiral nematic LC mixture that would enhance diffusion towards the focal point of the UV light source. ${ }^{[21]}$ Enhancing the phase separation in this way would also limit the concentration of non-crosslinked reactive monomer remaining between the polymer walls, which may polymerize upon exposure to ambient UV light (currently, all device characterization needs to be carried out in a dark room). Evidently, this is one important issue that needs to be addressed before the technique can be used to manufacture commercial devices. For example, in terms of preparing new optimised LC mixtures, careful consideration into the amount of monomer required to fabricate polymer walls of a precise volume, combined with additional monomers that could control the rate of diffusion towards the fabrication laser beam, should be taken into account. A more simple approach would involve washing out the remaining unpolymerized monomer in a solvent to form a ULH template that could subsequently be refilled with a chiral nematic LC. ${ }^{[22]}$ 

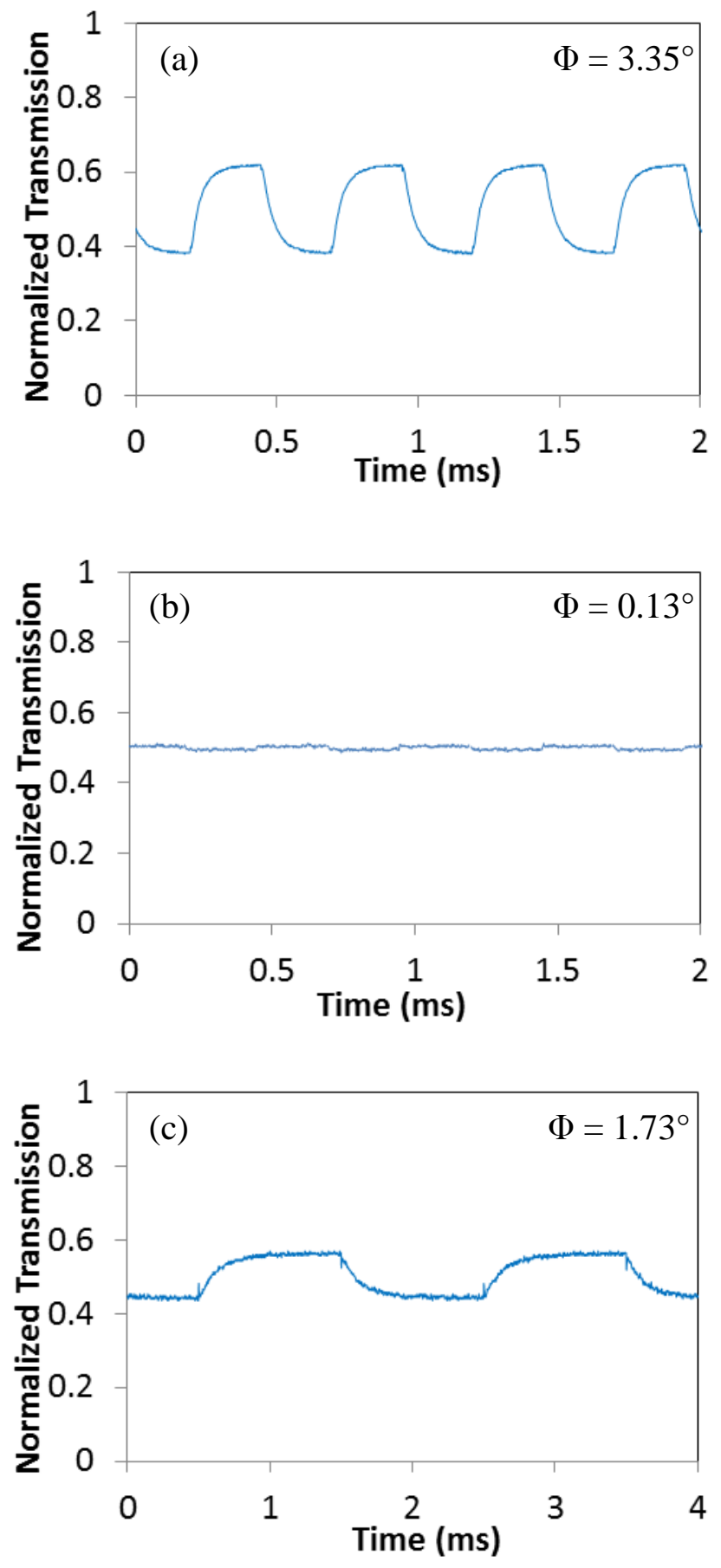

Figure 7. The electro-optic response of the three devices: (a) before polymerization; (b) after polymerization in the bulk (12 seconds exposure to a UV lamp at a distance of $10 \mathrm{~cm}$, emitting at $\lambda=365 \mathrm{~nm}$ and at a power density of $185 \mathrm{mWcm}^{-2}$ ); (c) with $5 \mu \mathrm{m}$ spaced polymer walls fabricated in-situ on the TPA set-up, under the application of a $\pm 27.5 \mathrm{~V}$ square wave. 


\section{Conclusion}

The spontaneous formation of a uniform lying helix alignment in a short pitch chiral nematic liquid crystal has been demonstrated through the in-situ fabrication of periodic polymer network structures using a two-photon absorption laser scanning lithography technique. Combined with a spatial light modulator that corrects for aberrations in the system, this fabrication technique allows the polymer structures to be written directly into a liquid crystal device. Polymer walls spaced at the order of the device thickness appear to optimise the uniform lying helix alignment and consequently lead to an increase in the flexoelectro-optic tilt angle relative to that obtained when polymerizing the bulk of the liquid crystal device. Investigations into the optimum materials for two-photon absorption processes could further enhance the device's electro-optic performance, for example, by limiting polymer diffusion to regions outside of the laser's focal spot. In principle, this technique can be employed to stabilise other inherently unstable liquid crystal states occurring in both the chiral and achiral nematic phase for displays applications or to engineer more complex multidimensional topological structures for a broader range of photonics applications.

\section{Acknowledgements}

The authors gratefully acknowledge financial support from the Engineering and Physical Sciences Research Council (UK) and Merck for an Industrial-CASE Studentship. SMM acknowledges The Royal Society for financial support.

\section{References}

${ }^{1}$ M. Hird, Liq. Cryst. 38, 11-12 (2011).

${ }^{2}$ S. T. Lagerwall, Mol. Crys. Liq. Crys. 543, 1 (2011).

${ }^{3}$ R. B. Meyer, Phys. Rev. Lett. 22, 918 (1969).

${ }^{4}$ J. S. Patel and R. B. Meyer, Phys. Rev. Lett. 58, 15 (1987). 
${ }^{5}$ N. Koma, T. Miyashita and T. Uchida, J. Soc. Info. Display 9, 8 (2005).

${ }^{6}$ P. Rudquist and S. Lagerwall, Flexoelectricity in Liquid Crystals, Imperial College Press, London, UK (2013).

${ }^{7}$ P. S. Salter, G. Carbone, S. A. Jewell, S. J. Elston and P. Raynes, Phys. Rev. E 80, 041707 (2009).

${ }^{8}$ P. Rudquist, L. Komitov and S. Lagerwall, Liq. Cryst. 24, 3 (1998).

${ }^{9}$ B. J. Broughton, M. J. Clarke, S. M. Morris, A. E. Blatch and H. J. Coles, J. Appl. Phys. 99, 023511 (2006).

${ }^{10}$ G. Carbone, D. Corbett, S. Elston, P. Raynes, A. Jesacher, R. Simmonds and M. Booth, Mol. Crys. Liq. Cryst. 544 (2011).

${ }^{11}$ R. B. Meyer and J. S. Patel, Flexoelectric liquid crystal device, US Patent 4917475 (1987).

${ }^{12}$ L. Komitov, G. Bryan-Brown, E. Wood and A. Smout, J. Appl. Phys. 86, 7 (1999).

${ }^{13}$ G. Carbone, P. Salter, S. Elston, P. Raynes, L. De Sio, S. Ferjani, G. Strangi, C. Umeton and R. Bartolino, Appl. Phys. Lett. 95, 011102 (2009).

${ }^{14}$ W. R. Zipfel, R. M. Williams and W. W. Webb, Nature Biotech. 21 (2003).

${ }^{15}$ P. Salter, A. Jesacher, J. Spring, B. Metcalf, N. Thomas-Peter, R. Simmonds, N. Langford, I. Walmsley and M. Booth, Opt. Lett. 37, 4 (2012).

${ }^{16}$ H. J. Coles, B. Musgrave, M. J. Coles and J. Willmott, J. Mater. Chem. 11 (2001).

${ }^{17}$ A. E. Blatch, M. J. Coles, B. Musgrave, and H. J. Coles, Mol. Crys. Liq. Crys. 401 (2003).

${ }^{18}$ H. J. Coles, M. J. Clarke, S. M. Morris, B. J. Broughton and A. E. Blatch, J. Appl. Phys. 99, 034104 (2006).

${ }^{19}$ H. Xianyu, S.-T. Wu and C.-L. Lin, Liq. Cryst. 36, 6-7 (2009)

${ }^{20}$ M. Mrukiewicz, P. Perkowski and K. Garbat, Liq. Cryst. 42, 7 (2015)

${ }^{21}$ R. Penterman, S. Klink, H. de Koning, G. Nisato and D. Broer, Nature 417 (2002).

${ }^{22}$ F. Castles, F. V. Day, S. M. Morris, D-H. Ko, D. J. Gardiner, M. M. Qasim, S. Nosheen, P. J.

W. Hands, S. S. Choi, R. H. Friend and H. J. Coles, Nature Mater. 11 (2012) 